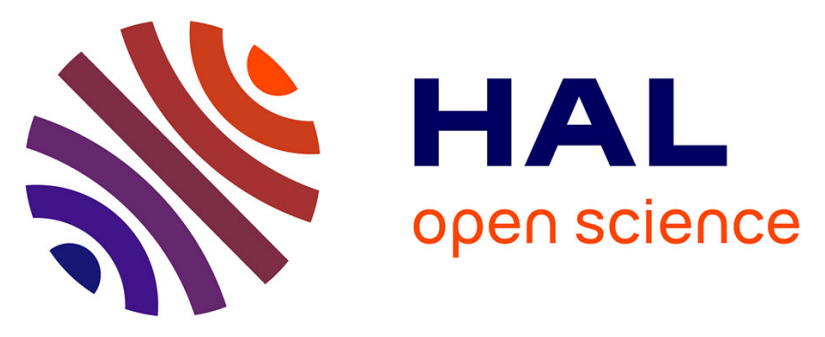

\title{
Measurement and Deembedding Technique for the on-wafer Characterization of Multiport Devices
}

Audrey Cayron, Christophe Viallon, Ayad Ghannam, Alessandro Magnani, Thierry Parra

\section{- To cite this version:}

Audrey Cayron, Christophe Viallon, Ayad Ghannam, Alessandro Magnani, Thierry Parra. Measurement and Deembedding Technique for the on-wafer Characterization of Multiport Devices. 2020 IEEE 20th Topical Meeting on Silicon Monolithic Integrated Circuits in RF Systems (SiRF), Jan 2020, San Antonio (TX), United States. pp.53-56, 10.1109/SIRF46766.2020.9040178 . hal-02921429

HAL Id: hal-02921429

https://hal.laas.fr/hal-02921429

Submitted on 25 Aug 2020

HAL is a multi-disciplinary open access archive for the deposit and dissemination of scientific research documents, whether they are published or not. The documents may come from teaching and research institutions in France or abroad, or from public or private research centers.
L'archive ouverte pluridisciplinaire HAL, est destinée au dépôt et à la diffusion de documents scientifiques de niveau recherche, publiés ou non, émanant des établissements d'enseignement et de recherche français ou étrangers, des laboratoires publics ou privés. 


\title{
Measurement and Deembedding Technique for the on-wafer Characterization of Multiport Devices
}

\author{
Audrey Cayron ${ }^{1}$, Christophe Viallon ${ }^{1}$, Ayad Ghannam ${ }^{2}$, Alessandro Magnani ${ }^{2}$ and Thierry Parra ${ }^{1,2}$ \\ ${ }^{1}$ LAAS-CNRS, University of Toulouse, CNRS, INSA, UPS, 7 avenue du Colonel Roche, F-31062, \\ Toulouse, France \\ 23DiS Technologies, 478 Rue de la Découverte, F-31670, Labège, France
}

\begin{abstract}
A useful technique is presented for on-wafer characterization of $n$-port devices, when measurement accesses are of unequal length and when a $m$-port vector network analyzer is used with $m<n$. The $n-m$ unused probing ports are left unterminated to simplify the measurement setup. The elimination of parasitic reflections arising from the unterminated ports and the deembedding of access contributions are performed from three measurements on two additional test patterns only. This method is demonstrated through the characterization up to $40 \mathrm{GHz}$ of an 8-port Butler matrix using a 4-port vector network analyzer.
\end{abstract}

Index Terms - Butler matrix, Deembedding, Millimeter waves, Multiport measurements, On wafer measurements, Port normalization, S-parameter measurement.

\section{INTRODUCTION}

The microwave characterization of a device on its own is an essential step before considering its implementation within a circuit or a system. However, difficulties to reach accurate performances can result from the test setup, especially when a multiport circuit is considered.

Without a specific and expensive $n$-port vector network analyzer (VNA), the full characterization of the $n$-port device is usually performed from several partial measurements involving a 2-port or a 4-port conventional VNA and matched loads as terminations of the remaining ports. From this methodology, the characterization of an $n$-port device using a $m$-port VNA ( $m$ is assumed to be even) with $m<n$, requires $n(2 n-m) / m^{2}$ partial measurements to acquire all the coefficients of the $n \times n$ scattering matrix.

However, this technique is not perfect and leads to measurement errors as it is impossible to present an ideal $50 \Omega$ termination on each unused port. Matched loads connected to RF probes bring some frequency-varying return losses, and lead to practical probing complexities while on-chip $50 \Omega$ terminations remain inaccurate and costly when such a solution is possible. Then, another solution that works very well with passive devices is to leave all unused ports unterminated, and to acquire a set of measurements whose values will be deliberately affected by the multiple reflection errors coming from these mismatched unterminated ports. These reflections lead to inconsistent $S$-parameters from a sub-matrix to another one, but the consistency can be recovered by considering the impedance of each port and by following several renormalization steps, as fully described in [1]. The resulting $S$-parameters then correspond to those that would have been measured if all ports were terminated with matched loads. To complete this method, the precise knowledge of the reflection coefficient at each unterminated port is essential for reaching accurate measurements [1]-[2]. In addition, when an on-wafer multiport device is to be characterized, those ports usually consist in Ground-Signal-Ground (GSG) or GSGSG pads connected to the DUT through access lines of different length. Then, several test patterns have to be included beside the DUT to evaluate the impedance provided by unterminated ports and to deembed the contribution of each access line.

This paper presents such a technique for the on-wafer characterization of multiport circuits, that minimizes the number of measurements as well as the number of required additional test patterns. The method is detailed in section II and it is applied in section III, for the on-wafer characterization of an 8-port Butler matrix, up to $40 \mathrm{GHz}$.

\section{Multiport Measurement TeChNiQue}

The proposed multiport characterization method is summarized in Fig. 1, when applied for the 8-port device of Fig. 2 (nevertheless, this method may be applied to any $n$-port device, regardless of $n$ ). Within this example, four different line lengths are used for connecting probing pads of the test fixture (labels " $P$ ") to inputs and outputs of the DUT (located into "Ref. Plane 2"). In these conditions, the $8 \times 8 S$-matrix of the DUT is reached from measurements of the six $4 \times 4$ submatrices, in addition with measurements performed on both lines of Fig. 3 .

Let $Z_{\text {open }}$ be the impedance of an unterminated pad, this impedance being seen from the "Ref. plane 1" location (Fig. 2). Considering this, the reference plane of all partial 4-port measurements has to be moved toward "Ref. plane $1 "$. This operation is carried out by deembedding the probing pads contributions. The $8 \times 8$ matrix cannot be 
reconstructed yet because the "Ref. plane 1 " is terminated by $Z_{\text {open }}$ at unused pads while being terminated by $50 \Omega$ at deembedded measurement ports. The reference impedance is thus inconsistent between sub-matrices. The next step then consists in the renormalization of the reference impedance of the 4-port sub-matrices from $50 \Omega$ to $Z_{\text {open }}$. It is performed by using the following expression [1]:

$$
\boldsymbol{S}^{\prime}=(\boldsymbol{I}-\boldsymbol{S})^{-1}(\boldsymbol{S}-\boldsymbol{T})(\boldsymbol{I}-\boldsymbol{S} . \boldsymbol{T})^{-1}(\boldsymbol{I}-\boldsymbol{S})
$$

where $\boldsymbol{S}$ is the original scattering matrix normalized to a given set of port impedances $\zeta_{i}(i=1$ to $n) . \boldsymbol{S}^{\prime}$ is the transformed scattering matrix normalized to a new set of port impedances $Z_{i}(i=1$ to $n)$. $\boldsymbol{I}$ is the $n \times n$ identity matrix. $\boldsymbol{T}$ is the $n \times n$ diagonal matrix containing the reflection coefficient $T_{i i}$ of the loads $Z_{i}$ referenced to the initial impedances $\zeta_{i}$. When renormalized relatively to $Z_{\text {open }}$, the six sub-matrices can be used to form the global $8 \times 8$ matrix of the DUT including access lines [1].

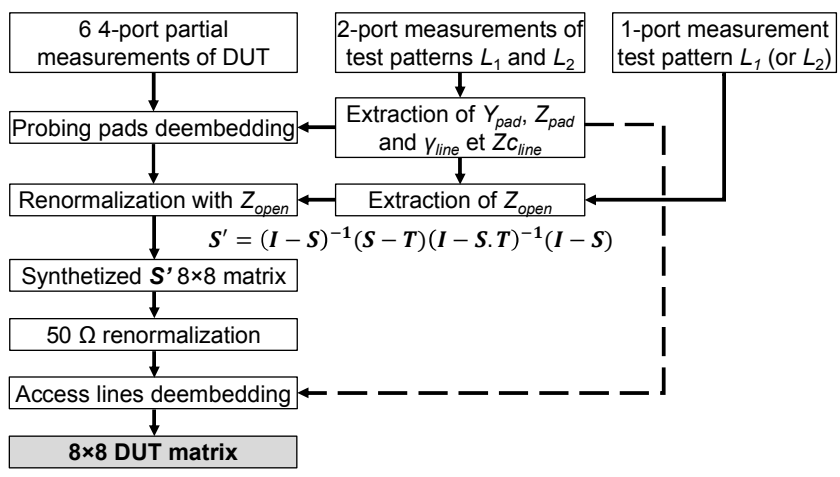

Fig. 1. Illustration of the deembedding procedure.

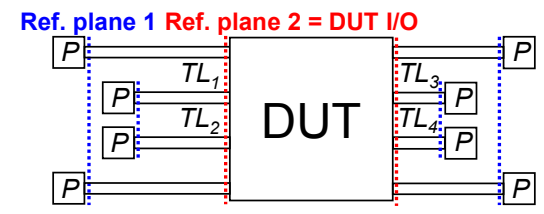

Fig. 2. Test fixture schematic for an 8-port DUT.

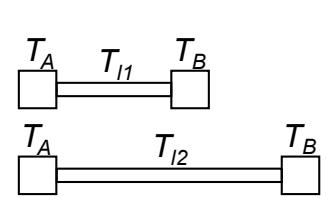

(a)

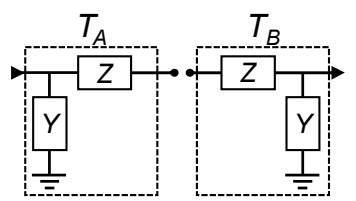

(b)
Fig. 3. (a) Schematic of the lines $L_{1}$ and $L_{2}$ including pads, and (b) lumped models of both pads.

Once the complete matrix of the circuit is built and renormalized back to $50 \Omega$ using (1), the influence of the access lines can be removed. This new operation of deembedding is carried out by using the additional measurements done on the transmission lines $L_{1}$ and $L_{2}$ defined in Fig. 3(a). Only two test patterns are required to perform all deembedding steps (i.e. removing the contribution of probing pads and of access lines). Both lines must exhibit the same characteristics than the ones interfacing the DUT, and they are used to extract the following parameters of the test fixture:

- propagation constant $\gamma$ and characteristic impedance $Z_{c}$, of the access transmission lines;

- the equivalent lumped-based pad model when the RF probe is in contact (Fig. 3(b));

- The impedance $Z_{\text {open }}$ provided by an unterminated pad.

The extraction of these parameters is described hereafter. The method is derived from [3].

First, the ABCD matrices $\boldsymbol{M}_{\mathbf{1}}$ and $\boldsymbol{M}_{\mathbf{2}}$ of the two lines are derived from measured scattering matrices, as follows:

$$
\begin{gathered}
\boldsymbol{M}_{\mathbf{1}}=\left(\begin{array}{ll}
m_{11} & m_{12} \\
m_{21} & m_{22}
\end{array}\right)=\boldsymbol{T}_{\boldsymbol{A}} \boldsymbol{T}_{\boldsymbol{l 1}} \boldsymbol{T}_{\boldsymbol{B}} \\
\boldsymbol{M}_{\mathbf{2}}=\left(\begin{array}{ll}
p_{11} & p_{12} \\
p_{21} & p_{22}
\end{array}\right)=\boldsymbol{T}_{\boldsymbol{A}} \boldsymbol{T}_{\boldsymbol{l} \mathbf{2}} \boldsymbol{T}_{\boldsymbol{B}}
\end{gathered}
$$

where $\boldsymbol{T}_{\boldsymbol{A}}$ and $\boldsymbol{T}_{\boldsymbol{B}}$ are the ABCD matrices of the two pads. These can be written as:

$$
\boldsymbol{T}_{\boldsymbol{A}}=\left(\begin{array}{cc}
1 & Z \\
Y & Y Z+1
\end{array}\right) \text { and } \boldsymbol{T}_{\boldsymbol{B}}=\left(\begin{array}{cc}
Y Z+1 & Z \\
Y & 1
\end{array}\right)
$$

Since both transmission lines differ only in length, they display both the same propagation constant $\gamma$ and characteristic impedance $Z_{c}$. Their respective matrix $\boldsymbol{T}_{\boldsymbol{l} \mathbf{1}}$ and $\boldsymbol{T}_{\boldsymbol{L} 2}$ are expressed as follows:

$$
\boldsymbol{T}_{\boldsymbol{l 1}(\mathbf{2})}=\left(\begin{array}{cc}
\cosh \left(\gamma l_{1(2)}\right) & Z_{c} \sinh \left(\gamma l_{1(2)}\right) \\
\frac{1}{Z_{c}} \sinh \left(\gamma l_{1(2)}\right) & \cosh \left(\gamma l_{1(2)}\right)
\end{array}\right)
$$

Both patterns are symmetric, so $m_{11}=m_{22}$ and $p_{11}=p_{22}$ are observed. By using this assumption, if we rewrite (2) and (3) in the form $\boldsymbol{M}_{\mathbf{1}(\mathbf{2})} \boldsymbol{T}_{\boldsymbol{B}}{ }^{-1}=\boldsymbol{T}_{\boldsymbol{A}} \boldsymbol{T}_{\boldsymbol{l 1}(\boldsymbol{l} 2)}$, and expand each term, we are able to derive the coefficients $m_{12}, m_{21}$, $p_{12}$ and $p_{21}$ as a function of $\boldsymbol{T}_{11}(\mathbf{l})$ and $\boldsymbol{T}_{\boldsymbol{A}(\boldsymbol{B})}$ matrix coefficients, as well as $m_{11}$ and $p_{11}$ values. The obtained expressions are rewritten in a matrix form as follows:

$$
\left(\begin{array}{ll}
m_{21} & m_{12} \\
p_{21} & p_{12}
\end{array}\right)=\boldsymbol{A}\left(\begin{array}{cc}
Y & \frac{Z}{1+Y Z} \\
\frac{1+Y Z}{Z_{c}} & \frac{Z_{c}}{1+Y Z}
\end{array}\right)
$$

where matrix $\boldsymbol{A}$ is defined as

$$
\boldsymbol{A}=\left(\begin{array}{ll}
\cosh \left(\gamma l_{1}\right)+m_{11} & \sinh \left(\gamma l_{1}\right) \\
\cosh \left(\gamma l_{2}\right)+p_{11} & \sinh \left(\gamma l_{2}\right)
\end{array}\right)
$$

The expression (6) is solved for $Y, Z$ and $Z_{c}$ by inverting the matrix $\boldsymbol{A}$ and knowing $\boldsymbol{M}_{\mathbf{1}}$ and $\boldsymbol{M}_{\mathbf{2}}$. The propagation constant $\gamma$ is extracted beforehand from $\boldsymbol{M}_{\mathbf{1}}$ and $\boldsymbol{M}_{\mathbf{2}}$ by using the method proposed in [4]. 
The impedance $Z_{\text {open }}$ of an unconnected probing pad is assessed as follows. Let $Z_{\text {meas }}$ be the 1-port measurement of test pattern $L_{1}$ (or $L_{2}$ ). $Z_{\text {open }}$ is then the termination impedance of a 2-port network constituted by the probing pad modeled by $Y$ and $Z$ followed by the transmission line of length $l_{1}$ characterized by $Z_{c}$ and $\gamma$. This 2-port network is expressed by the ABCD matrix $\boldsymbol{T}$ :

$$
\boldsymbol{T}=\boldsymbol{T}_{\boldsymbol{A}} \boldsymbol{T}_{\boldsymbol{l 1}}=\left(\begin{array}{ll}
t_{11} & t_{12} \\
t_{21} & t_{22}
\end{array}\right)
$$

$Z_{\text {open }}$ is retrieved by removing the contribution of the 2-port network $\boldsymbol{T}$ from $\mathrm{Z}_{\text {meas }}$ data:

$$
Z_{\text {open }}=\frac{t_{22} Z_{\text {meas }}-t_{12}}{t_{11}-t_{21} Z_{\text {meas }}}
$$

\section{RESULT ON A 8-PORT BUTLER MATRIX}

The method is applied to the characterization of the 8-port 4x4 Butler matrix described in Fig. 4. The circuit has been designed to address $5 \mathrm{G}$ beamforming applications in the $25-29 \mathrm{GHz}$ frequency range. In theory, insertion losses of $6 \mathrm{~dB}$ are observed from input ports 1 to 4 toward output ports 5 to 8 . A phase shift $\Delta \varphi$ of $-45^{\circ}, 135^{\circ},-135^{\circ}$ and $45^{\circ}$ between outputs is obtained when the excitation source is switched from port 1 to 4 , respectively.

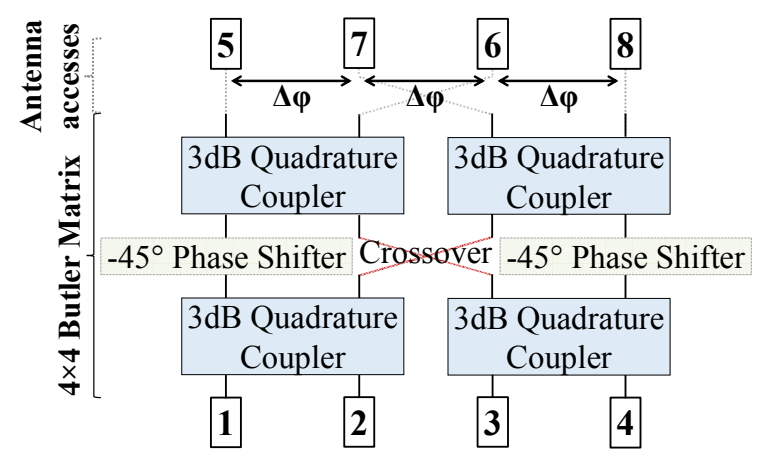

Fig. 4. Block diagram of a $4 \times 4$ Butler Matrix.

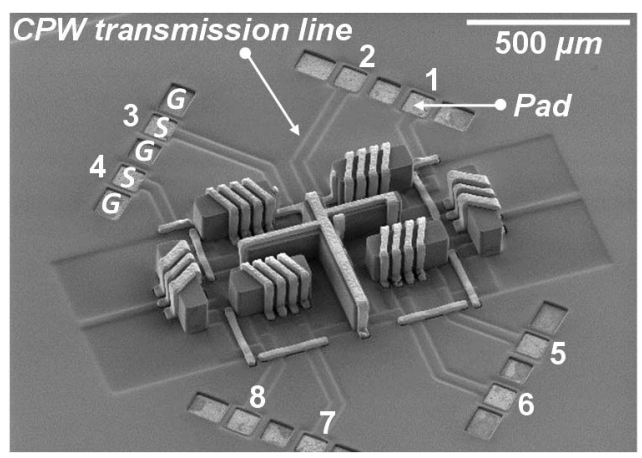

Fig. 5. SEM picture of the 8-port Butler matrix.
The Butler matrix, shown in Fig. 5, has been fabricated using a dedicated technology that enables the manufacture of 3-D solenoids [5]. The eight internal ports of the Butler matrix are connected to dual ground-signal-ground (GSGSG) coplanar pads through CPW lines of different length. These CPW lines have also been integrated next to the circuit through two test structures of $400 \mu \mathrm{m}$ and $1600 \mu \mathrm{m}$-length in order to implement the aforementioned method. The measurements have been performed using a Keysight N5247A PNA-X 4-port vector network analyzer (VNA) from $10 \mathrm{GHz}$ to $40 \mathrm{GHz}$ using several $\mathrm{GSG}$ and GSGSG $150 \mu \mathrm{m}$ Cascade Infinity RF probes.
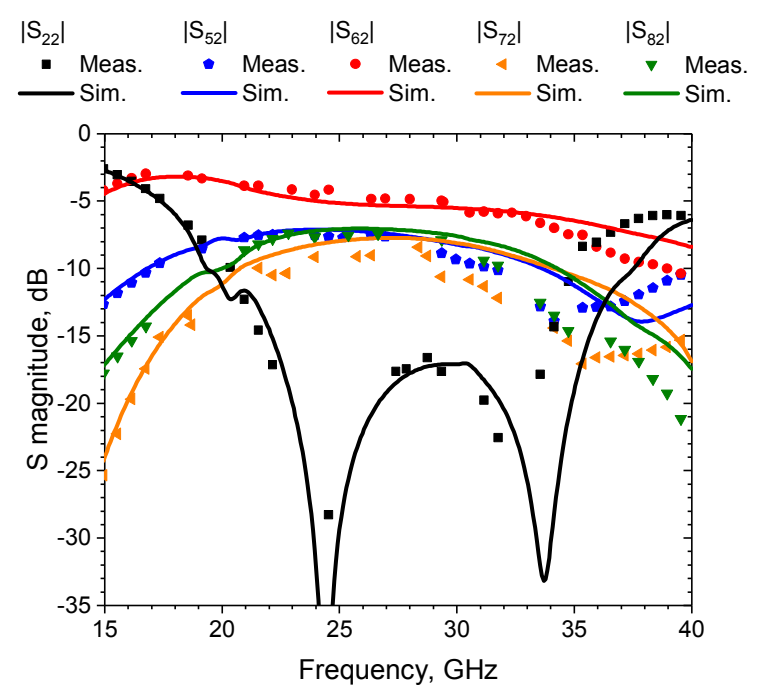

Fig. 6. Recovered Butler matrix (dots) and simulated (full lines) transmissions between port 2 and the output ports 5 to 8 .

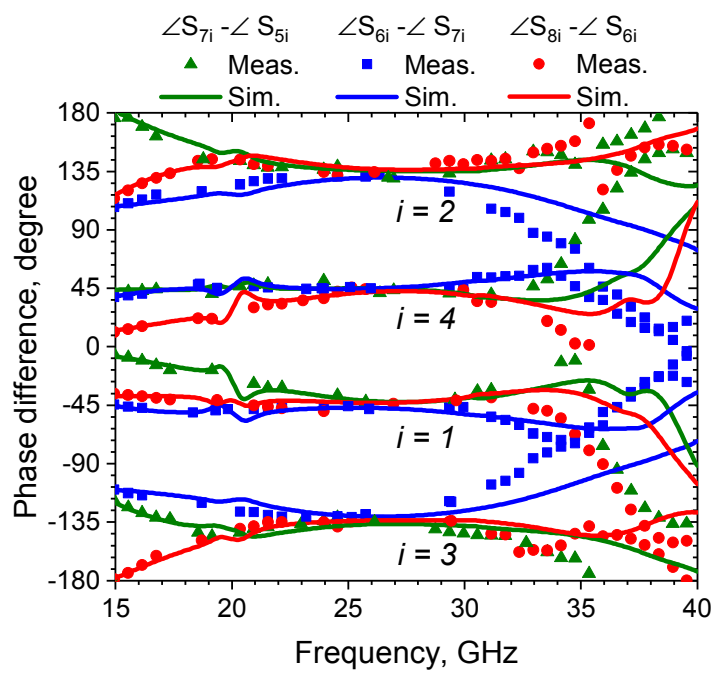

Fig. 7. Recovered Butler matrix (dots) and simulated (full lines) phase differences between outputs 7-5, 6-7 and 8-6 for an excitation applied at port $i(i=1$ to 4$)$. 
The deembedded measured performances of the circuit are plotted in Fig. 6 and Fig. 7 for the case of an excitation source applied on port 2. One can notice the good agreement between measurements and electromagnetic simulations. From 15 to $28.5 \mathrm{GHz}$, the differences are kept below $1.3 \mathrm{~dB}$ for insertion losses and $8^{\circ}$ for phase differences. However, the measured bandwidth of the circuit is slightly reduced from the high frequency side for some of the parameters $\left(S_{52}, S_{72}, S_{82}\right.$ and $\left.S_{22}\right)$. For them, the frequency shift is of $1.5 \mathrm{GHz}$ starting at $29 \mathrm{GHz}$. It may be traced to a process variation on the copper lines fabrication modifying interwire capacitors values.

Finally, it can be noticed that the points representing the measurements in Fig. 6 and Fig. 7 are not regularly spaced according to the frequency. From these results, we have effectively removed all data parts identified as aberrations in order to properly compare the measurements with simulations. Fig. 8 shows these aberrations on the parameters $S_{62}$ and $S_{22}$. At some frequencies, the results deviate from what could be expected and lead to several unphysical peaks. This problem originates from the matrix inversions needed in the renormalization expression (1). It has been known for many years and has not yet found a completely satisfactory solution [6]-[9].

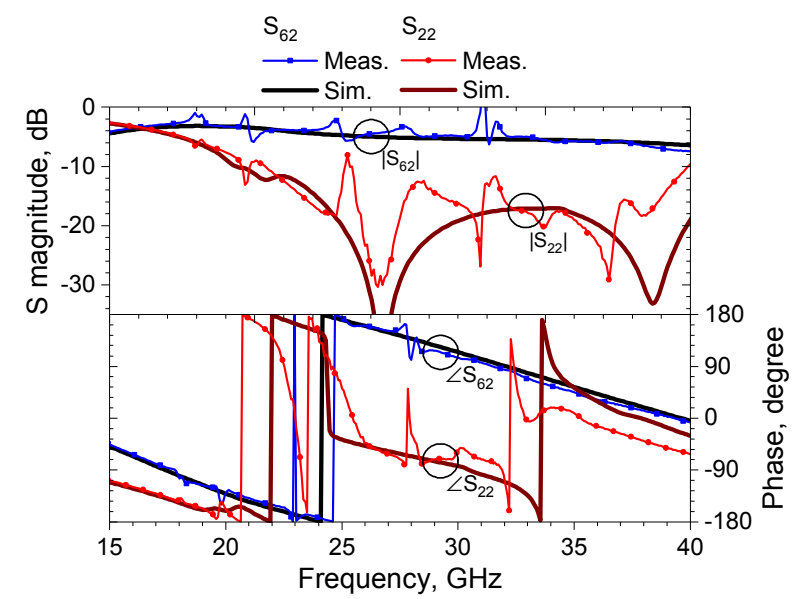

Fig. 8. Magnitude and angles of $S_{62}$ and $S_{22}$ parameters to illustrate the numerical instabilities caused by the renormalization process.

The amplitude and periodicity of these numerical perturbations mainly depend on the reflection coefficient of auxiliary terminations in conjunction with measurement errors. In addition, lower the devices losses, higher the impact of this dispersions on device performance. A smoothing procedure as suggested in [7] could improve accuracy on results. [9] proposed another measurement procedure that assigns a variable reflecting coefficient at one port of the device to minimizes numerical instability. Based on the work of Chen [6]-[9], we plan to investigate a procedure that could minimize these numerical perturbations.

\section{CONCLUSION}

This paper proposes a method to characterize a linear $n$-port device with a $m$-port VNA (with $m<n$ ). The $n \times n$ S-matrix of the device is built from $n(2 n-m) / m^{2} m \times m$ sub-measurements. As a significant simplification, because the number of measurements to be considered, all the ports of the DUT that are not connected to the VNA are left open. Moreover, by adding only three extra measurements on two 2-port test patterns (transmission lines of different lengths) it becomes possible to deembed access lines of unequal lengths. The method is finally applied to the on-wafer characterization of an 8-port Butler matrix. The good agreement observed between simulations and measurements validates the entire methodology.

\section{REFERENCES}

[1] J. C. Tippet, et al., «A Rigorous Technique for Measuring the Scattering Matrix of a Multiport Device with a 2-Port Network Analyzer », IEEE Trans. Microw. Theory and Techn., vol. 30, $\mathrm{n}^{\circ} 5$, p. 661-666, 1982.

[2] D. G. Kam, et al., « Multiport Measurement Method Using a TwoPort Network Analyzer With Remaining Ports Unterminated », IEEE Microw. Wireless Comp. Lett., vol. 17, nº 9, p. 694-696, 2007.

[3] J. E. Zúñiga-Juárez, et al. "An improved two-tier L-L method for characterizing symmetrical microwave test fixtures," Measurement, vol. 44, no. 9, pp. 1491-1498, 2011.

[4] J. A. Reynoso-Hernandez, et al. "Computation of the Wave Propagation Constant $\gamma$ in Broadband Uniform Millimeter Wave Transmission Line," in Asia Pacific Microw. Conf., Singapore, Singapore, 1999, pp. 266-269.

[5] A. Ghannam, et al., "Ultra-Compact, High-Performance, 3D-IPD Integrated Using Conformal 3D Interconnects", in IEEE $68^{\text {th }}$ Electronic Components and Technology Conference ECTC, San Diego, CA, USA, 2018, pp. 1082-1088.

[6] C.-J. Chen, et al., "An Error Analysis of the Scattering Matrix Renormalization Transform", IEEE Trans. Microw. Theory and Techn., vol. 57, no. 4, pp. 863-868, 2009.

[7] C.-J. Chen, et al., "Measurement of Noncoaxial Multiport Devices Up to the Intrinsic Ports", IEEE Trans. Microw. Theory and Techn., vol. 57, no. 5, pp. 1230-1236, 2009.

[8] C.-J. Chen, et al., "Accuracy Criterion for S-Matrix Reconstruction Transforms on Multiport Network", IEEE Trans. Microw. Theory and Techn., vol. 59, no. 9, pp. 2331-2339, 2011.

[9] C.-J. Chen, et al., "Multiport Network Measurement using a ThreePort VNA", in Asia-Pacific Microwave Conference APMC, Seoul, Korea (South), 2013, pp. 636-638. 